\title{
Abschiebungshaft und irreguläre Migration
}

\section{Martin Hagenmaier}

\section{Einleitung}

$\mathrm{D}$ ie massenhafte Abschiebungshaft im Schengenraum zeigt offene Politik- und Rechtsfragen der Verhältnismäßigkeit und der Zuständigkeit für irreguläre Migration an. Sie bleibt ein Symptom von Zuwanderungspolitik ohne Ordnung und Perspektive. Nach spektakulären und aufregenden Berichten von Wanderungen biblischen Ausmaßes und halsbrecherischen Überquerungsversuchen des Mittelmeeres und von politischen Abwehrversuchen Europas verschwindet das Problem im Alltag und wird nicht weiter verfolgt. ${ }^{1}$ Die Migrationsversuche enden häufig in Abschiebungsbemühungen der Zielländer. Das besondere Symptom dieser Bemühungen ist die Abschiebungshaft. Hier treffen vorwiegend Männer aus allen Abwanderungsländern zusammen und sehen ihrer „Rückführung“ entgegen. Der Grund der Rückführung ist die Einreise ohne Papiere oder mit falschen Papieren, wobei nicht der ursprüngliche Migrationsversuch, sondern das Herumreisen in Europa auf der Suche nach einem Platz zum Bleiben immer häufiger die Ursache von Abschiebungsbemühungen wird.

Innerhalb Europas differieren die zulässigen Abschiebungshaftzeiten zwischen 30 Tagen in Frankreich und unbegrenzt in den Niederlanden, Dänemark, Schweden und Großbritannien. Auch die Modalitäten der Haftprüfung sind unterschiedlich. Während in den Niederlanden die Inhaftierung durch die Polizei erfolgt und dann von Amts wegen eine monatliche Überprüfung stattfinden muss, kann in Deutschland eine Abschiebungshaft von bis zu sechs Monaten ausgesprochen werden, die um höchstens zwölf Monate verlängert werden kann ( $\$ 62$ AufenthG). Zur Verlängerung ist hier jeweils ein neu begründeter Antrag der antragsberechtigten Behörde - Ausländeramt oder Bundespolizei - notwendig. Die meisten Beschlüsse zur Abschiebungshaft umfassen den Zeitraum von drei Monaten. Die Länder des Schengen-Dublin-Raumes haben in Asyl- und Migrationsfragen ihre Zuständigkeiten miteinander abgesprochen und Regeln des Umganges damit vereinbart. ${ }^{2}$ Das Ergebnis ist an den Problemen mit der Abschiebungshaft zu betrachten.

\section{Eine „Europageschichte"}

Acht Jahre war R. in Italien, eingereist als Bürger Tunesiens. Dort hat er zunächst mit seinen gültigen Papieren mit Visum gearbeitet und das verdiente Geld nach Hause geschickt. Als das Visum abgelaufen war, ,versäumte' er, rechtzeitig ein neues zu beantragen. Das ging so lange gut, bis der Pass auch abgelaufen war. Dann wollte keinen neuen beantragen. Die tunesischen Behörden sollten keine Kenntnis von seinem Aufenthalt in Italien erhalten, wahrscheinlich wegen seiner Vorgeschichte. Nach acht Jahren, in denen er in ganz Italien herumkam, wurde er verhaftet. Einen Grund für diese Verhaftung nannte er nicht. Offenbar hing es mit einer Gesetzesübertretung und/ oder fehlenden Papieren zusammen. Jedenfalls verfügte ein Gericht seine Ausweisung und verzichtete aus diesem Grund auf eine Verurteilung. Die entspräche nach deutschem Recht einem Vorgehen nach § 154b III StPO (Absehen von Klageerhebung bei Ausweisung) oder nach 456a I StPO (Absehen von der Vollstreckung wegen Ausweisung). Eine Abschiebung erfolgte offenbar nicht, so dass R. sich seine Reiseroute selbst aussuchen konnte. Statt nach Tunesien ging er in die Schweiz. Dort saß er nach Entdeckung seiner illegalen Einreise sechs Monate in Abschiebungshaft. Inzwischen gab er an, aus Algerien zu stammen. Da aus Algerien keine Papiere zu bekommen waren, wurde er aus der Abschiebungshaft entlassen mit der Auflage, das Land zu verlassen. So kam er nach Belgien, wo er Asyl beantragte. Dort lebte er zwei Jahre bei einer Belgierin, die er aber nicht heiraten wollte, obwohl sie ihm das anbot. Als diese Beziehung zu Ende war und der Asylantrag abgelehnt, kam er wiederum vier Monate in Abschiebungshaft, wurde jedoch aus demselben Grund wie in der Schweiz wieder entlassen. Erneut erhielt er eine Aufforderung, das Land zu verlassen. Die nächste Station waren naheliegend: die Niederlande. Dort wurde er sehr schnell als illegal eingereister Ausländer festgenommen und verbrachte acht Monate in Abschiebungshaft. Papiere aus Algerien konnten auch hier nicht beschafft werden, was wiederum zur Entlassung in die Freiheit führte mit der Aufforderung, das Land zu verlassen. Auf der Reise nach Skandinavien wurde er an der dänischen Grenze festgenommen. Vier Monate Abschiebungshaft folgten in Deutschland. Im Verlauf dieser Abschiebungshaft konnte ermittelt werden, dass sein Herkunftsland Tunesien ist. Wer diese Tatsache aufdeckte, ist unbekannt. Möglicherweise gab es eher zufällige Hinweise aus genauerer Kenntnis der verschiedenen Einfärbungen der arabischen Sprache mit einem folgenden „Blindversuch“ der Ausländerbehörde.

Als dies bekannt war, erzählte R. den Hintergrund seiner lang dauernden europäischen Flucht. Er hatte in Tunesien offenbar ein aus seiner Sicht durch Machtverhältnisse und nicht durch Tatsachen herbeigeführtes Urteil eines Gerichts zu erwarten oder dieses war bereits ergangen. Das war der Grund seiner zunächst, legalen Flucht'. Die Illegalisierung war sein eigenes Werk, wurde aber auch durch die europäischen Verhältnisse provoziert. Als seine Verwandtschaft zu Hause ihm versichert hatte, die Strafe würde nicht mehr vollzogen, weil inzwischen zwei Amnestien verkündet worden seien, entschloss er sich zur Mitarbeit bei der Rückführung. Diese erfolgte innerhalb von zwei Wochen - nach rund zwölfjähriger Migrationsgeschichte.

In gut zwölf Jahren in Europa kamen in diesem Fall zweiundzwanzig Monate Abschiebungshaft in vier verschiedenen Ländern zusammen, dreimal mit in Kauf genommener oder beabsichtigter Illegalisierung des Migranten.

Weitere Beispiele aus Gruppengesprächen mit Abschiebungshäftlingen

\section{a) Eine Reise aus Europa nach Europa}

Kosovo - Albanien - Durres - Bari. Dort hat ihn die italienische Polizei kontrolliert. Sie haben gesagt, er müsse das Land verlassen. Dann gaben sie ihm „einen Stempel für sechs Stunden“. „Was willst $\mathrm{Du}$, ...gehst du nach Deutschland...", soll die italienische Polizei zu ihm gesagt haben. Das kann auch Einbildung sein....

Er hat danach in der Schweiz Asyl beantragt. Er blieb dort bis zur Aufforderung zur Ausreise. Danach reiste er nach Holland. Dort blieb er ebenfalls bis zur Ausreiseaufforderung. Die nächste Station war Schweden. Von dort reiste er nach Deutschland, weil ihn offenbar die schwedischen Behörden nach Italien zurückschieben wollten. Dort, so beteuert er, hat er nie einen Asylantrag gestellt. „Warum soll ich dann dorthin zurück?", heißt seine Frage.

Im Kosovo könne er nicht leben. Die Probleme seien zu groß. Näheres über die Probleme schildert er auch nicht auf Nachfrage. Er war schon in Kroatien und in Slowenien. Da kann er doch auch problemlos reisen. Warum geht das in Europa sonst nicht? Schweden hat eine Übernahme abgelehnt. Die Abschiebungshaft verlängert sich so erheblich, weil jetzt die Anfrage in Italien abgewartet werden muss. (Alter: Mitte 20) 


\section{b) Iran}

Ein Mann aus dem Iran kommt aus Schweden oder Norwegen oder .... Er soll nach Griechenland zurückgeschickt werden. Dort hat er zweieinhalb Jahre gelebt - illegal. Danach beantragte er in diversen Ländern Asyl, zuletzt in Norwegen. Von dort reiste er nach Deutschland, weil der Asylantrag in Norwegen abgelehnt worden war und eine Abschiebung nach Griechenland anstand. (Alter: Ende 20)

\section{c) Makedonien}

Übermorgen fliegt einer nach Skopje. Seine Frau war mit den drei Kindern nach Dänemark zurückgeschickt worden, obwohl sie alle zusammen 4 Jahre in Deutschland waren. Sie sind von Dänemark bereits nach Skopje abgeschoben worden und warten dort auf ihn. Sie haben kein Haus und keine Wohnung. Aber sie haben Verwandte. Das wird eng! Er kann nicht verstehen, dass man ihn von seiner Familie getrennt hat. (Alter: knapp 40)

\section{d) Nigeria}

Ich bin nach dreizehn Jahren Deutschland immer noch auf der Abschussliste. Jetzt versuchen sie seit elf Monaten mich loszuwerden. Aber sie schaffen es nicht. Zuerst haben sie mich in Berlin sechs Monate eingesperrt. Dann hat mich das Obergericht freigelassen. Sie gaben mir ein Ticket nach Kiel. In Kiel gab mir das Ausländeramt eine Fahrkarte nach Berlin, dort soll ich bei meiner nigerianischen Botschaft einen Ausweis oder ein tc (traveler certificate) holen. Die in der Botschaft haben gesagt: ,Was, du bist dreizehn Jahre in Deutschland. Dann geben wir dir keine Papiere. Kämpf hier für deine Rechte!' Da bin ich nach Kiel zurückgefahren. Unterwegs wurde ich kontrolliert. Das war in Brandenburg. Da kam ich nach Eisenhüttenstadt in die Abschiebungshaft. Auch von dort haben sie mich wieder entlassen. Danach war ich in Hamburg unterwegs. Daher kam ich in Abschiebungshaft in Hamburg, in der UHA und in Fuhlsbüttel. Da sie mich nicht abschieben konnten, versuchen sie es jetzt hier in Rendsburg.

Irgendwann einmal erwarte ich Gerechtigkeit. Ich habe niemand etwas getan. Sieben Jahre war ich mit einer deutschen Frau verheiratet. Wir haben ein Kind. Das ist jetzt neun Jahre alt. Nach der Scheidung sollte ich sofort ausreisen, obwohl es für geschiedene Ehepartner von Deutschen nach drei Jahren ein eigenständiges Aufenthaltsrecht gibt. Dann bin ich ein bisschen in die USA gereist. Als ich wiederkam, wurde ich auf dem Flughafen festgenommen. Ich hatte nicht die richtigen Papiere. Ich habe dann erklärt, dass ich doch schon so lange in
Deutschland gelebt hatte. „Das gilt jetzt nicht mehr", sagten sie. Dann haben sie mich nach Kiel geschickt. Eigentlich wollte ich doch mein Kind sehen. Doch die Mutter hat sich dagegen gewehrt. Ich bin zurück nach Nigeria, auf eigene Kosten und ganz allein. Als ich wiederkam, sagten sie: „Was willst du hier, wo kommst du her? Das geht aber nicht." Schließlich bekam ich eine Duldung. Ich habe jetzt eine neue Freundin und ein fünf Monate altes Kind. Das gilt auch nicht. Erst wenn das Kind zwei Jahre alt ist, dann hat es ein Anrecht auf Umgang mit dem Vater oder so. Ich will aber doch Vater sein.... Ich bin auch nicht kriminell. Nur einmal wurde ich verurteilt. Das war gleich am Anfang. Da bin ich mit meinem nigerianischen Führerschein Auto gefahren. Das wurde als „Fahren ohne Führerschein" vom Gericht verurteilt.

In Nigeria arbeiten viele deutsche Leute. Soll man die auch ins Gefängnis bringen? Oder was soll man mit ihnen machen? Ich versteh nicht, warum Deutschland das tut. (Alter: Mitte 30)

\section{e) Elfenbeinküste.}

Er ist seit vierzehn Jahren in Deutschland. 1992 beantragte er Asyl und wurde abgelehnt, erhielt jedoch eine Duldung. 2002 heiratete er eine deutsche Frau. Die Ehe ging nicht so gut. Wenn er viel zur Arbeit ging, um Geld zu verdienen, dann sagte seine Frau: ,Du bist nie zu Hause.' Wenn er dagegen dann auf die Kinder aufpasste, war es auch nicht recht. Er wollte nicht wie ein Bettler bei den Schwiegereltern das Geld abholen, um mit der Familie zu leben. Daher folgte die Trennung und ein Wiederbeginn, dann wieder eine Trennung. Nun ist er doch geschieden. Die Dauer der Ehe war nicht ganz zwei Jahre. Das reicht nicht für das eigenständige Aufenthaltsrecht.

Als er mit siebzehn aus der Elfenbeinküste wegging, hatte das den folgenden Grund: Die Leute machen nichts als „Scheiße“. Die Banditen kommen in die Stadt und suchen sich die herumstreunenden Jungs zusammen. Dann geben sie ihnen Waffen und sagen: Macht das oder das kaputt...!. Dann schlagen die alles kaputt und schießen einfach auf alles. Das ist schrecklich. Ich würde gerne wieder nach Hause gehen, wenn das alles aufhört. Aber ich glaube, die können das nicht. Das ist in Afrika überall so. Hier sind Jungen mit zwölf oder vierzehn Jahren Kinder. Dort werden sie für alles eingesetzt. Sie bekommen ein wenig Geld und dann machen sie alles.

\section{f) Algerien}

Im Alter von dreizehn Jahren ist er mit zwei anderen Jungen aus Algerien abgehauen. Wie man das einfach mal so macht, das erzählte er bisher nicht. Sie kamen zum Fährhafen nach Marokko. Dann sind sie auf einen Lastwagen gesprungen, der gerade auf die Fähre fuhr. Sie wurden nicht entdeckt und kamen nach Spanien. Seither ist er in ganz Europa unterwegs. Auf die Frage, wie viele Asylanträge er gestellt hat, zählte er nach einigem Nachdenken sechs Länder auf und sagte dann: „Überall!“ Wenn er irgendwo abgelehnt wurde, ging er einfach in das nächste Land. Aktuell kommt er jetzt aus Schweden. Dort war er in der Abschiebungshaft. Die ist nicht so schwer bewacht. Daher konnte er weggehen und einen neuen Versuch unternehmen. Eigentlich wollte er nach Frankreich. Bei einer Kontrolle wurde er in Schleswig-Holstein festgenommen. Die Anfrage auf Rückübernahme läuft. Empörend findet er, dass die Polizei ihm sein Geld weggenommen hat. Er hat es höchstpersönlich verdient. Das waren über 1700 Euro.

Jetzt ist er 28 und hat in Algerien „nichts zu suchen". Seine Eltern sind geschieden und haben beide neue Familien und viele Kinder. Die wollen beide mit ihm nichts mehr zu tun haben. Die Scheidung ist auch der Grund, weshalb er weggegangen ist. Auf die Frage, ob er nicht doch einen Versuch mit einem Neuanfang mit Hilfe eines oder beider Eltern starten könnte, antwortet er: „Die sagen, er sei doch jetzt erwachsen, er müsse selber sehen, wie er weiterkommt."

Wenn er jetzt die Möglichkeit hat, dann geht er nach Afghanistan, sagt er mit Blick auf seinen Mitgefangenen, der von dort kommt. Was er dort will, kann er ziemlich genau beantworten: Vielleicht gibt es da was zu kämpfen oder man fliegt einfach in die Luft. Das sagt er mit entsprechenden Bewegungen. Und sein afghanischer Nachbar fügt hinzu: ,Die Deutschen haben nichts $\mathrm{zu}$ befürchten. Die haben bei uns einen guten Ruf. Aber die anderen, die USA und die Engländer und die Spanier, die haben es ja schon gezeigt bekommen. Das ist uns ein Bedürfnis, uns zu wehren. Nicht gegen die guten Leute wie Euch!'

Die Reihe der Beispiele lässt sich beliebig verlängern. Sie weisen alle dieselben Merkmale auf:

1) Die Umstände des Beginns von Migrationsversuchen sind so vielfältig wie die Menschen, die sie unternehmen und wie die Umstände, aus denen sie kommen. Die Migration beginnt bisweilen als Reise, oft aber unter irregulären Umständen wozu u.a. falsche Papiere und Reisehelfer (Schlepper) und manchmal mehrere tausend Euro gehören können. Auch Zufälle spielen eine Rolle.

2) In vielen Fällen werden nach der Ablehnung einer Aufenthaltserlaubnis mehrere Länder durchquert oder in mehreren Ländern Asylanträge gestellt. Dadurch verlängert sich die 
Zeit der Aufenthalte außerhalb des Herkunftslandes immer weiter.

3) Ausreisefristen verstreichen, ohne dass eine Ausreise erfolgt bzw. die Abschiebung möglich gewesen wäre.

4) Die nationalen Behörden streiten um die $\mathrm{Zu-}$ ständigkeit und nehmen sie jeweils nur widerwillig wahr.

5) Einfache Lösungen bestehen in der Inhaftierung und der anschließenden Ausweisung ohne weitere behördliche Aktivität mangels eines rücknahmebereiten Abschiebungsziellandes. So entsteht eine bewusst in Kauf genommene bzw. herbeigeführte Illegalität. Alle Möglichkeiten des Überlebens werden offiziell durch diesen Akt gestrichen.

6) Abschiebungshaft dient in dreißig bis fünfzig Prozent der Fälle der Abklärung von europäischer Zuständigkeit. In weiteren zehn Prozent der Fälle kann diese nicht geklärt werden. Dadurch wird das aktuelle Aufenthaltsland zuständig.

7) Die behördlichen Bemühungen sind nicht in der Lage, das Problem zu lösen. Sie verschieben es nur bis hin zur Illegalisierung.

\section{Bürokratische Verwahrlosung}

Die Hilflosigkeit bzw. Ineffektivität europäischer Bürokratie in diesem Bereich darf mit gutem Recht bürokratische Verwahrlosung Europas genannt werden. Besonders eindrucksvoll wird diese Verwahrlosung durch das Verhalten nach erfolglosen Recherchen zur Herkunft belegt. Trotz der Schengener Verträge und der Dubliner Übereinkommen verweisen Teilnehmer dieser Verträge Menschen ohne Papiere aus ihren Ländern. Nach geltendem Recht ist das untersagt, weil niemand von Amts wegen illegal gemacht werden darf. Um aber eine Zuständigkeit zu vermeiden, passiert das häufiger. Damit geschieht genau das, was nach den Verträgen eigentlich zu vermeiden wäre.

Die Schengen- und Dublinabkommen wurden u.a. als Lösung für die Verwaltung von Migration in Europa geschlossen. Die Wirklichkeit verhält sich anders. Das Konstrukt versagt mindestens bei der irregulären Migration. Es regelt nur die Verantwortlichkeit nach einer umständlichen Prüfung auf das Prinzip „erster Asylantrag“. Geregelt wird aber nicht der Umgang mit der Aufenthaltsbeendigung. Hier arbeitet jedes Land nach eigenen Gesetzen. Manche Länder wollen sich offenbar der Verantwortung für eine Abschiebung oder die Übernahme bei fehlender Abschiebungsmöglichkeit nicht stellen, sondern nötigen Menschen, die sie nicht akzeptieren, durch Entzug von Duldungen etc. zur irregulären Weiterwanderung in das nächste europäische Land. Damit wirken Schengen und Dublin als Verwaltungsordnung mit dem Ergebnis (des Versuchs) der Verhinderung der Übernahme von Verantwortung.
Die Anfragen auf Rückübernahme von illegal eingereisten Migranten und Migrantinnen (irreguläre Migration ist ein eher männliches Phänomen) setzt einen Verwaltungsakt ohnegleichen in Gang. Für Deutschland bittet die Zentrale im Bundesamt für Migration über die europäische Zentrale in Straßburg das möglicher Weise zuständige Land förmlich um eine Übernahme der illegal eingereisten Person. Die Rückantwort erfolgt nach förmlicher Prüfung auf demselben Weg. Obwohl das Verfahren per e-mail abgewickelt wird, dauern die Antworten bis zu mehreren Monaten oder bleiben ganz aus. Wo früher zwischen Deutschland und Skandinavien der ,kleine Grenzverkehr' die Angelegenheit in fünf Minuten bis zu einer halben Stunde regeln konnte, kommt heute die Antwort aus Stockholm nach vier Wochen. Bis dann ein vorgeschriebener Reiseweg (meistens per Flug) gebucht und verabredet ist, können nochmals mehrere Wochen verstreichen. Das gilt für fast alle Nachbarschaftsgrenzen in Europa. Vor allem bei Skandinavien kommt noch dazu, dass nahezu alle als, illegal' von dort eingereisten Personen eine Asylidentifikationskarte mit sich führen, die ihren Status ,beweist'. Da diese aber kein gültiges Reisepapier ist, kann es auch nicht zur Ausreise verwendet werden.

Ganz nüchtern kann also festgestellt werden, dass in Deutschland und anderen europäischen Ländern Menschen zwar aufgrund von nicht erlaubter Einreise oder nicht erlaubtem Aufenthalt festgehalten und in Haft genommen werden. In der Hälfte der Fälle ist diese Haft jedoch eine ,Vorhaltungshaft' und keine Abschiebungshaft. Die Verwaltungen klären wochenlang die Zuständigkeit. Bis ein Ergebnis erarbeitet ist, bleibt der Betroffene in Haft. In Zeiten des elektronischen Datenverkehrs und der sofortigen Identifikationsmöglichkeiten jedes einzelnen Migranten per ,eurodac' bereits durch die Bundespolizei (also noch vor dem Antrag auf Abschiebungshaft) darf diese Inhaftierungspraxis als unangemessen oder gar als schikanös betrachtet werden, wenn sie überhaupt noch rechtskonform ist.

Man muss auch damit rechnen, dass sich irreguläre Migranten auf solche bürokratische Verwahrlosung eingestellt haben, sie einkalkulieren und in ihrem Sinne nutzen. Zunächst bedeutet Abschiebungshaft zumindest eine einigermaßen angemessene Versorgung mit Nahrung sowie medizinischer und anderer Betreuung. Sie enthebt für einige Wochen der Unsicherheit der Illegalität und gibt die Möglichkeit, seinen Fall noch einmal amtlich und rechtlich prüfen zu lassen. Und sie erweist vielleicht die Unmöglichkeit von Abschiebung. Das System gibt die Möglichkeiten, durch Reisen in jeweils neue Zuständigkeitsbereiche zu kommen, die dann umständlich mit den bisherigen verhandeln müssen. Statt einer Abschiebung kommt es zur Zurückschiebung, da- mit das zuständige Land die Abschiebung vornimmt.

In den meisten Fällen stellt die Abschiebungshaft die Aussichtslosigkeit der Migrationsbemühungen vor Augen. Darunter leiden am schwersten Familienväter, die alleine anstelle ihrer Familie inhaftiert werden. (Eine etwas makabre Art von Stellvertretung.) Dass hier sogar noch Steigerungen möglich sind, zeigt das Beispiel c) aus Makedonien. Hier wurde die Zuständigkeit für Frau und Kinder von der für den Familienvater europäisch getrennt. Im Falle ganzer Familien wäre eine Verhaftung am ehesten $\mathrm{zu}$ vermeiden, weil selbst die Behörden davon ausgehen, dass Familien in solchen Situationen zusammenhalten.

\section{Migrationshintergründe und europäische Zu- ständigkeit}

Die Hintergründe irregulärer Migration und die Motive zum Migrationsversuch sind so vielfältig wie die beteiligten Individuen. Die hier vorgestellten Beispiele sind nicht repräsentativ, aber doch typisch. Die Migrationsversuche beginnen bei Männern bereits im jugendlichen Alter und entstehen aus einer subjektiven Bewertung der eigenen Situation im Leben. Der dreizehnjährige Junge, der sich aus Algerien davon macht, ist durchaus keine Ausnahme. Er erklärt dieses „Weglaufen“ mit der Scheidung seiner Eltern, wodurch sein Platz im Leben zerstört wird.

Der Mann aus der Elfenbeinküste bringt die Anomie - Situation seines Herkunftslandes treffend auf den Punkt und klärt gleichzeitig über politische Vorgänge auf, die aus europäischer Sicht unverständlich sind. „Herumlungernde“ Jungen werden von Banditen benutzt, um Unruhe anzuzetteln sowie Angst und Not zu verbreiten. Das schafft die für ihre Geschäfte und Bandenherrschaft notwendigen Verhältnisse. Wer kann, geht aus diesen Verhältnissen weg. Ungerecht empfundene Justiz, manchmal auch die Angst vor gerecht(fertigt)er Verfolgung durch dieselbe, stellt einen weiteren Grund für den Versuch dar, aus dem eigenen Land zu verschwinden.

„Die Probleme sind zu groß, daher kann ich in meinem Heimatland nicht leben. "So lautet häufiger die Begründung für irreguläre Migration aus den Nachfolgestaaten von Jugoslawien. In diesem Fall bleibt es bei dieser Auskunft. In anderen ist die Rede von Erpressungen und Nötigungen durch die Mafia bzw. durch Nachfolgeorganisationen der albanischen UCK, von wirtschaftlicher Aussichtslosigkeit aufgrund fehlender Arbeit und von Diskriminierung im Falle der Zugehörigkeit zu Minderheiten.

Jede von einem Individuum und/oder seiner 
Umwelt als aussichtslos, gefährlich, ungerecht oder als erhebliche Benachteiligung interpretierte Lebenssituation kann dazu führen, Migrationsversuche irregulärer Art zu unternehmen. In den Zielstaaten - so der Umkehrschluss - erscheint offenbar das Leben leichter, chancenreicher, gerechter und weniger gefährlich. Der Zugang zu den Zielstaaten ist jedoch erheblich reglementiert oder wie in den meisten Fällen nicht möglich. Daher bilden sich andere Wege der Einreise heraus, die den formalen Gesetzen zuwiderlaufen, indem Visa und Identitäten gekauft bzw. fingiert werden oder die Reise einfach ohne Papiere erfolgt. Die Zielstaaten verweigern gerade Menschen aus Ländern mit insuffizienten, ungerechten und anomischen Strukturen verstärkt und mit Absicht die Teilnahme an ihren Ressourcen. Die Migrierenden kennen auch die Ausrichtung der erstrebten gesellschaftlichen Sicherheit, die es erschwert, Menschen ohne Papiere abzuschieben. Aus diesen Konstellationen entsteht der Kreislauf irregulärer Migration mit all ihren Folgen und der bürokratischen Verwahrlosung Europas.

Die auf dem Wege der Abschiebung zurückgeschafften Migranten erhalten neben Einreiseverboten für diese Maßnahmen eine Rechnung, die sie nie mit Mitteln ihrer Herkunftsregion bezahlen können. Sie schulden dem Abschiebungsstaat nach mehrwöchiger Abschiebungshaft und den notwendigen Transporten Geld in Höhe mehrerer Jahreseinkommen. Damit geraten die Betroffenen in weitere unlösbare Situationen. Die Rechnungen sind höher als die der Schleuser. Zur Deckung von zu erwartenden Unkosten werden Barmittel der Abzuschiebenden eingezogen (gedeckt durch § 66 I, § 67 I Nr. 1-3, § 67 III AufenthG), was diese als ungerechtfertigte Willkür empfinden.

Europa tut sich mit der formalisierten bürokratischen nationalen Zuständigkeitslösung keinen Gefallen im Hinblick auf irreguläre Migranten. Es fördert im Gegenteil die Zirkulation von irregulärer Migration durch die einzelnen Staaten der EU. Sinnvoller und gerechter wäre es, eine gemeinsame europäische Migrationsplattform mit einem Ausweis zu schaffen, damit eine europäische Zuständigkeit entsteht, die den Umgang mit irregulärer Migration zusammenfasst und erleichtert. Damit würde mindestens die Hälfte aller Abschiebungshaftanordnungen unnötig.

Um jedoch der irregulären Migration nicht noch Nahrung zuzuführen, müssten die Einund Ausreisemöglichkeiten so formuliert werden, dass eine einsichtige und berechtigte Chance auf Aufenthalt oder Einwanderung auch für Menschen besteht, die sich in ihrem Land benachteiligt fühlen und einen anderen Platz zum Leben suchen. Es darf sich nicht lohnen, ohne Papiere zu reisen, vielmehr sollte die reguläre Reise durch bessere Vorbereitungsmög- lichkeiten im Herkunftsland belohnt werden. Wer dann noch ohne Papiere reist, kann mit Sicherheit der Verschleierungs- und Betrugsabsicht geziehen und schnellstmöglich zurückgeschickt werden. Oder es handelt sich um einen echten Asylfall mit dem Hintergrund einer überstürzten und unvorhergesehenen Flucht.

\section{Arbeit und irreguläre Migration}

Die Migranten aus dem ersten sowie aus den Beispielen d) (indirekt), e) und f) lassen ein Bild von ihrer „Arbeit" unter irregulären Migrationsbedingungen erkennen. Der Tunesier aus Italien hat zunächst reguläre Arbeit, bis er sich selbst illegal macht. Bei d) erscheint im Umkehrschluss, dass der Betroffene regulär gearbeitet hat. Er vergleicht sich mit den Deutschen, die in Nigeria arbeiten. Der Unterschied scheint zu sein, dass bei den Deutschen in Nigeria die Aufenthaltsfrage nicht problematisch ist. Auch dort wird vermutlich die Arbeitsmöglichkeit oder -erlaubnis vom Aufenthaltsstatus abhängen. In Deutschland jedenfalls dominiert der Aufenthaltsstatus alle Fragen des Arbeitsmarktes, weil die Arbeitserlaubnis dem Status nachgeordnet ist. Allerdings gehen Aufenthaltsmöglichkeiten besser $\mathrm{zu}$ ordnen, wenn ein Arbeitsvertrag bereits vor der Einreise in Aussicht steht. E) berichtet über Arbeit, wobei er im nicht aufgezeichneten Gesprächsteil noch hinzufügte, er mache jede Arbeit, die er bekommen könne. Hier hängt die Arbeit mit ,Gemütslagen' in der Familie zusammen. Ein interessanter Hinweis ergibt sich aus der Bewertung von Arbeit. Sie gibt ihm die Möglichkeit, „nicht wie ein Bettler bei den Schwiegereltern das Geld ab(zu)holen". Arbeit bewertet e) als Chance, ein geachteter selbstverantwortlicher Mann zu sein, der nicht betteln muss. Er stellt ein Männerbild vor, bei dem ein Mann in der Lage sein sollte, „seine Familie zu ernähren“. Unter anderem scheint das einer der leitenden Gründe auch für die irreguläre Migration zu sein. F) ist empört, dass ihm sein selbstverdientes Geld weggenommen wird. Er muss also in seinem letzten Aufenthaltsland Schweden irgendeiner Arbeit nach gegangen sein. Das bedeutet, dass er jedenfalls nicht auf staatliche Unterstützung gebaut oder sich etwas „dazuverdient" hat. Der Betroffene versuchte, sich eigene Erwerbsquellen zu erschließen. Zu der Art der Arbeit gibt er in dieser Fragestellung keine Auskunft.

Das Geldverdienen durch Arbeit ist eine Hauptabsicht auch der irregulären Migranten. Die erste Zielsetzung ist nicht das Unterkommen in den Sozialsystemen in Europa. „Nicht wie ein Bettler dazustehen", das gehört zum Männerbild und damit zu den kulturellen Zielsetzungen der meisten Migranten. Politik und Recht sollten damit endlich offensiver umgehen. Das erscheint jedoch nur möglich, wenn die politische Diskussion nicht mehr an unseli- gen Alternativen wie „Flüchtling“ versus „Wirtschaftsmigrant", sondern an Realitätswahrnehmungen ansetzt. Um Realität wahrzunehmen, ist es sinnvoll, sich die absurden politischen Verhältnisse in vielen Teilen dieser Welt und die Anomie in den wirtschaftlichen Verhältnissen innerhalb und zwischen den Staaten und Staatenbünden anzuschauen. Dazu muss man neben den Nachrichten aus aller Welt auch die Geschichten der Migranten anhören. ${ }^{3}$

Der Verfasser ist Gefängnisseelsorger in der JVA Kiel.

\section{Fußnoten}

1 Stellvertretend sei hier genannt: Ansturm der Armen, Titel des Nachrichtenmagzins Der Spiegel, Nr. 26 vom 26.6.2006, 66-91. Als zentrale Geschichte wird hier die schließlich zum Einwanderungserfolg führende vierjährige Reise eines Migranten aus Ghana nach Spanien nachgestellt und als Folie für die Geschichte innerafrikanischer gegenseitiger Ausbeutungs-, Unterdrückungs- und Gewaltverhältnisse genutzt. Garniert ist dies mit afrikanischen Einsichten aus der Sicht eines Afrikaners, der es nach Europa geschafft hat. Sein Migrationsgrund waren 1000 Dollar Schulden in Accra. (75). Sonja Margolina, Absurder Verschleiß. Miserable Integration: Deutschland ist kein Einwanderungsland, Süddeutsche Zeitung 62 . Jahrgang, Nr. 150 vom 3. 7. 2006, 13. Hier steht eine Geschichte irregulärer Migration aus der Ukraine im Zentrum, die die Absurdität der ausländerrechtlichen Regelungen unterstreicht. Migrationshintergrund ist hier eine problematisch-tragische Familiensituation. Die Autorin spricht von einem „absurden Verschleiß an Humankapital", vor allem im Hinblick auf das Arbeitsverbot. Sie diagnostiziert einen Mangel an pragmatischer Orientierung an eigenen (nationalen gesellschaftlichen) Interessen, wie es überall auf der Welt normal sei. Den Grund erblickt sie in der aus dem Zweiten Weltkrieg und dem Holocoust abgeleiteten Viktimisierung von Migranten in Deutschland. Das könnte zu der These führen, dass eine vorwiegend weltanschaulich-ethische Orientierung in bestimmten Fällen die Lösung von Problemen verhindert, statt Menschen zu schützen.

2 Mitteilung der Kommission an den Rat und an das Europäische Parlament über eine Gemeinschaftspolitik zur Rückkehr illegal aufhältiger Personen, KOM(2002)564 endgültig, Brüssel, 14.10.2002

VERORDNUNG (EG) Nr. 343/2003 DES RATES vom 18. Februar 2003 zur Festlegung der Kriterien und Verfahren zur Bestimmung des Mitgliedstaats, der für die Prüfung eines von einem Drittstaatsangehörigen in einem Mitgliedstaat gestellten Asylantrags zuständig ist, veröffentlicht im Amtsblatt der Europäischen Union L50/1 vom 25. 2. 2003.

VERORDNUNG (EG) Nr. 1560/2003 DER KOMMISSION vom 2. September 2003 mit Durchführungsbestimmungen zur Verordnung (EG) Nr. 343/2003 des Rates zur Festlegung der Kriterien und Verfahren zur Bestimmung des Mitgliedstaats, der für die Prüfung eines von einem Drittstaatsangehörigen in einem Mitgliedstaat gestellten Asylantrags zuständig ist, veröffentlicht im Amtsblatt der Europäischen Union.

3 Beispielsweise: Maack, Rüdiger Nur die Starken überleben, Über die desolate Situation illegaler Migranten in Afrika, Deutlandradio am 10.07.2006, · 18:40 Uhr. 